\title{
Template bank for spinning compact binary mergers in the second observation run of Advanced LIGO and the first observation run of Advanced Virgo
}

Debnandini Mukherjee $\odot,{ }^{1,2,3, *}$ Sarah Caudill,,${ }^{1,4, \dagger}$ Ryan Magee,${ }^{3,5}$ Cody Messick,,${ }^{3,5,6}$ Stephen Privitera, ${ }^{7}$ Surabhi Sachdev, ${ }^{8,3}$ Kent Blackburn, ${ }^{8}$ Patrick Brady, ${ }^{1}$ Patrick Brockill, ${ }^{1}$ Kipp Cannon, ${ }^{9,10}$ Sydney J. Chamberlin, ${ }^{3,5}$ Deep Chatterjee, ${ }^{1,11,12}$ Jolien D. E. Creighton, ${ }^{1}$ Heather Fong, ${ }^{9,10}$ Patrick Godwin, ${ }^{3,5}$ Chad Hanna, ${ }^{3,13,5}$ Shasvath Kapadia, ${ }^{1,14}$ Ryan N. Lang, ${ }^{15,16}$ Tjonnie G. F. Li, ${ }^{17}$ Rico K. L. Lo, ${ }^{17,8}$ Duncan Meacher, ${ }^{18,1}$ Alex Pace, ${ }^{18}$ Laleh Sadeghian, ${ }^{1}$ Leo Tsukada, ${ }^{10,19}$ Leslie Wade, ${ }^{20}$ Madeline Wade, ${ }^{20}$ Alan Weinstein, ${ }^{8}$ and Liting Xiao ${ }^{8}$

${ }^{1}$ Leonard E. Parker Center for Gravitation, Cosmology, and Astrophysics,

University of Wisconsin-Milwaukee, Milwaukee, Wisconsin 53201, USA

${ }^{2}$ Department of Physics, Indian Institute of Technology Bombay, Powai, Mumbai 400 076, India

${ }^{3}$ Department of Physics, The Pennsylvania State University, University Park, Pennsylvania 16802, USA

${ }^{4}$ Nikhef, Science Park, 1098 XG Amsterdam, Netherlands

${ }^{5}$ Institute for Gravitation and the Cosmos, The Pennsylvania State University, University Park, Pennsylvania 16802, USA

${ }^{6}$ Department of Physics, University of Texas, Austin, TX 78712, USA

${ }^{7}$ Albert-Einstein-Institut, Max-Planck-Institut für Gravitationsphysik, D-14476 Potsdam-Golm, Germany

${ }^{8}$ LIGO Laboratory, California Institute of Technology, MS 100-36, Pasadena, California 91125, USA

${ }^{9}$ Canadian Institute for Theoretical Astrophysics, 60 St. George Street, University of Toronto, Toronto, Ontario M5S 3H8, Canada

${ }^{10}$ RESCEU, The University of Tokyo, Tokyo 113-0033, Japan

${ }^{11}$ Center for Astrophysical Surveys, National Center for Supercomputing Applications, Urbana, IL, 61801, USA

${ }^{12}$ Illinois Center for Advanced Studies of the Universe, Department of Physics, University of Illinois at Urbana-Champaign, Urbana, IL 61801, USA

${ }^{13}$ Department of Astronomy and Astrophysics, The Pennsylvania State University, University Park, Pennsylvania 16802, USA

${ }^{14}$ International Centre for Theoretical Sciences, Tata Institute of Fundamental Research, Bengaluru 560089, India

${ }^{15}$ Hillsdale College, Hillsdale, Michigan 49242, USA

${ }^{16}$ Massachusetts Institute of Technology, Cambridge, MA 02139, USA

${ }^{17}$ Department of Physics, The Chinese University of Hong Kong, Shatin, New Territories, Hong Kong

${ }^{18}$ The Pennsylvania State University, University Park, Pennsylvania 16802, USA

${ }^{19}$ Department of Physics, Graduate School of Science, The University of Tokyo, Tokyo 113-0033, Japan

${ }^{20}$ Department of Physics, Hayes Hall, Kenyon College, Gambier, Ohio 43022, USA

(Received 19 December 2018; accepted 23 February 2021; published 26 April 2021)

We describe the methods used to construct the aligned-spin template bank of gravitational waveforms used by the Gstreamer and Ligo Algorithm Library (GstLAL)-based pipeline to analyze data from the second observing run of Advanced Laser Interferometer Gravitational Wave Observatory (LIGO) and the first observing run of Advanced Virgo. The bank expands upon the parameter space covered during Advanced LIGO's first observing run, including coverage for merging compact binary systems with total mass between $2 \mathrm{M}_{\odot}$ and $400 \mathrm{M}_{\odot}$ and mass ratios between 1 and 97.988 . Thus the systems targeted include merging neutron star-neutron star systems, neutron star-black hole binaries, and black hole-black hole binaries expanding into the intermediate-mass range. Component masses less than $2 \mathrm{M}_{\odot}$ have allowed (anti-)aligned spins between \pm 0.05 , while component masses greater than $2 \mathrm{M}_{\odot}$ have allowed (anti-) aligned between \pm 0.999 . The bank placement technique combines a stochastic method with a new gridbank method to better isolate noisy templates, resulting in a total of 677,000 templates.

DOI: $10.1103 /$ PhysRevD.103.084047

\footnotetext{
*debnandini.mukherjee@ligo.org

†sarah.caudill@ligo.org
} 


\section{INTRODUCTION}

The first observing run (O1) of the Advanced Laser Interferometer Gravitational Wave Observatory (LIGO) [1,2] detectors collected data from September 12, 2015 to January 19, 2016, during which two gravitationalwave $(\mathrm{GW})$ signals were detected at greater than $5 \sigma$, GW150914 [3] and GW151226 [4] from the mergers of two binary black hole (BBH) systems. The second observing run $(\mathrm{O} 2)$ of Advanced LIGO ran from November 30, 2016 to August 26, 2017, with Advanced Virgo [5] joining the run for the month of August. By the end of the $\mathrm{O} 2$, GWs from a total of ten binary black hole mergers had been observed across the $\mathrm{O} 1$ and $\mathrm{O} 2$ [6-9]. In addition, the low-latency discovery of GWs from a binary neutron star (BNS) merger [10] was also reported from the O2.

These types of signals are targeted by all-sky matchedfilter-based searches including GstLAL [11-16], PyCBC [17-20] and multi-band template analysis pipeline [21]. Matched-filter-based searches correlate detector data with waveforms predicted by general relativity drawn from a template bank. The template bank contains waveforms covering a multidimensional parameter space of component masses and spins. If a template closely matches a hidden signal in the data, then a peak (or trigger) in the correlation time series will be produced. The search pipelines then employ a number of techniques to ensure that triggers are found in operating detectors within the intersite propagation time and that the signal has the expected morphology and amplitude.

Such sources are also targeted by unmodeled searches that do not use template waveforms, for example, the coherent WaveBurst algorithm [22]. Such searches are especially effective for the heavier mass binaries, which have shorter template waveforms within the detector frequency band and hence look similar to short duration noise transients or glitches, making it difficult for them to be recovered by the template based searches. The unmodeled searches, requiring minimal assumptions about the waveform, have so far been more sensitive to the heavier binaries [23]. They are also expected to be more sensitive to signals from such heavier binaries, including nonfundamental higher order mode effects, than some matched-filter based searches [24]. Also, techniques to construct template banks directly from numerical relativity simulations have also been explored, as described in [25]. In this paper we will focus on the GstLAL-based inspiral search.

The GstLAL-based inspiral search pipeline (henceforth referred to as GstLAL) operates in two modes, a lowlatency online mode and an off-line deep-search mode. In this paper, we describe both the template bank used in the online mode for issuing low-latency alerts to astronomy partners, as well as the template bank used in the off-line mode for the deep analysis of the $\mathrm{O} 2$ data.

During the O1, the matched-filter-based searches of РyСBC and GstLAL used a common template bank with total masses between $2 \mathrm{M}_{\odot}$ and $100 \mathrm{M}_{\odot}[26,27]$ to search for stellar-mass binary black holes. For the O2, separate banks were constructed and utilized [28] to enhance the independence of search-pipeline results. Additionally, for the O1, a separate search for GWs from intermediate-mass black hole binaries (IMBHBs) was performed using a template bank with total mass between $50 \mathrm{M}_{\odot}$ and $600 \mathrm{M}_{\odot}$ [29]. The IMBHB search thus partially overlapped the stellar-mass search in the mass range between $50 \mathrm{M}_{\odot}$ and $100 \mathrm{M}_{\odot}$. With increase in the search sensitivities in the $\mathrm{O} 2$, the stellar-mass search could be expanded to include the IMBHB region. Hence, for the $\mathrm{O} 2$, an integrated search was implemented with a template bank covering up to a total mass of $400 \mathrm{M}_{\odot}$.

The paper is organized in the following way. In Sec. II we describe the design and construction of the integrated template bank used by GstLAL for the analysis of the $\mathrm{O} 2$ data. In Sec. III we describe the performance of the bank in recovering simulated signals from a variety of astrophysical populations. We present our conclusions in Sec. IV.

\section{DESIGN AND CONSTRUCTION OF THE O2 BANK}

\section{A. Astrophysical source targets}

The O2 GstLAL search targeted GW signals from merging binary compact objects with component masses between $1 \mathrm{M}_{\odot}$ and $399 \mathrm{M}_{\odot}$. These include binary systems with two neutron stars (BNS), two black holes (BBH), or a neutron star and a black hole (NSBH). This component mass region is known to be populated with compact objects produced from the collapse of massive stars. With stellar evolution models, neutron stars can form in the mass range between $1 \mathrm{M}_{\odot}$ and $3 \mathrm{M}_{\odot}$ [30-34], although, there is only one observed neutron star with a mass larger than $2 \mathrm{M}_{\odot}$ [35] and those in binaries do not approach $2 \mathrm{M}_{\odot}$ [36]. Stellar evolution models also predict that black holes may exist with a minimum mass down to $2 \mathrm{M}_{\odot}$ [37] and a maximum mass up to $100 \mathrm{M}_{\odot}$ or potentially higher $[38,39]$. Black holes with masses between $\sim 100 \mathrm{M}_{\odot}$ and $\sim 10^{5} \mathrm{M}_{\odot}$ are classified as intermediate-mass black holes and could have formed through hierarchical merging of lower mass black holes [40]. This search is also sensitive to GWs from binaries of primordial black holes (PBH), formed from over-dense regions in the early Universe. However, distinguishing a PBH GW signal from a conventional stellar-evolution black hole GW signal would not be possible with this search and is, instead, pursued in a separate search of the subsolar mass region [41].

We also define different ranges of allowed angular momentum for component neutron stars and component black holes. We consider the dimensionless spin parameter $\chi=c|\vec{S}| / G m^{2}$, where $\vec{S}$ is the angular momentum and $m$ is the component mass. Observations of the fastest spinning pulsar constrain $\chi \lesssim 0.4$ [42], while pulsars in binaries 


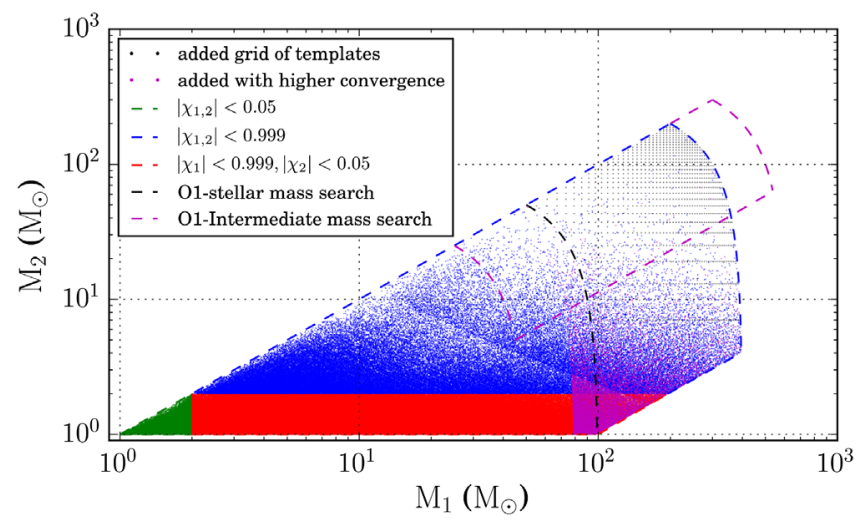

FIG. 1. The template bank used by the $\mathrm{O} 2 \mathrm{GstLAL}$ off-line search in component mass space. The templates representing the different astrophysical populations are shown in green for BNS, blue for $\mathrm{BBH}$, and red for $\mathrm{NSBH}$.

have $\chi \leq 0.04$ [43]. X-ray observations of accreting BHs indicate a broad distribution of BH spins [44-46], while the relativistic Kerr bound $\chi \leq 1$ gives the theoretical constraint [47].

These observations and evolution models inform the ranges of parameters we define for our template banks. As shown in Fig. 1, we can see the BNS, NSBH, and BBH populations represented in the $\mathrm{O} 2 \mathrm{GstLAL}$ off-line search. We impose an additional constraint on the component dimensionless spins of template waveforms by requiring their orientations to be aligned or antialigned with the orbital angular momentum of the binary $\hat{L}$. Then the dimensionless projections of the component spins along $\hat{L}$ are defined as $\chi_{i} \equiv c\left|\vec{S}_{i} \cdot \hat{L}\right| / G m_{i}^{2}$. The region in green marks the BNS templates with component masses between $1 \mathrm{M}_{\odot}$ and $2 \mathrm{M}_{\odot}$ and (anti-)aligned dimensionless spin magnitudes with $\chi_{1,2}<0.05$. This $\chi$ limit is motivated by the observational limit of $\chi \leq 0.04$ but with some added uncertainty. The region in blue marks the $\mathrm{BBH}$ templates with component masses between $2 \mathrm{M}_{\odot}$ and $399 \mathrm{M}_{\odot}$, mass

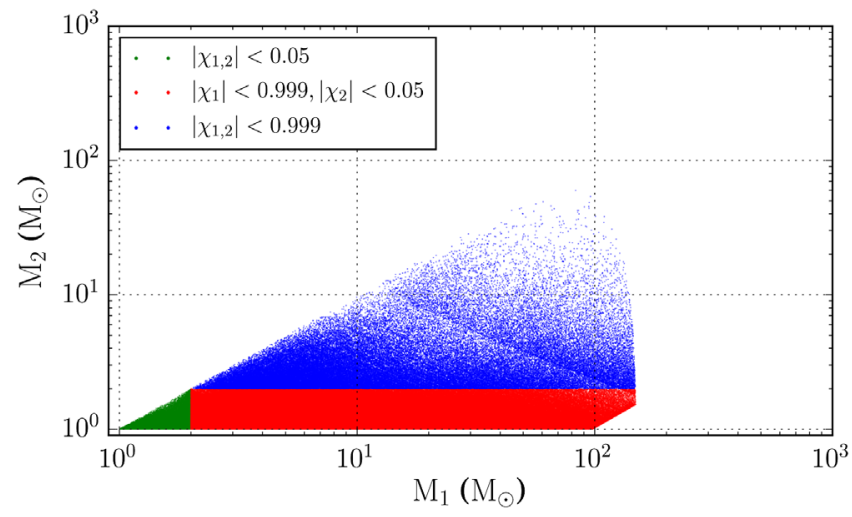

FIG. 2. The template bank used by the $\mathrm{O} 2$ GstLAL online search in component mass space. The templates representing the different astrophysical populations are shown in green for BNS, blue for $\mathrm{BBH}$, and red for NSBH. ratios greater than 1 and less than 98.988, which is the maximum for the bank and (anti-)aligned dimensionless spin magnitudes with $\chi_{1,2}<0.999$. This $\chi$ limit is chosen to be as close to the theoretical limit of 1 as possible with current waveform approximants, as described in Sec. II B 2. The templates in red mark the NSBH range with the neutron star mass between $1 \mathrm{M}_{\odot}$ and $2 \mathrm{M}_{\odot}$ and the black hole mass between $2 \mathrm{M}_{\odot}$ and $200 \mathrm{M}_{\odot}$. For these systems, neutron stars have $\chi_{1,2}<0.05$ and black holes have $\chi_{1,2}<0.999$.

In Fig. 2, we can see the BNS, NSBH, and BBH populations represented in the $\mathrm{O} 2 \mathrm{GstLAL}$ online search. The BNS templates cover the same component mass and dimensionless spin magnitude range as the off-line bank. However, an additional restriction employed in total mass $\left(M>150 \mathrm{M}_{\odot}\right)$ resulted in different component mass ranges for $\mathrm{NSBH}$ and $\mathrm{BBH}$ templates for the online template bank. The maximum allowed total mass is $150 \mathrm{M}_{\odot}$ to remove high-mass templates, which correspond to short waveforms that recover short transient noise fluctuations (glitches) at a high rate.

\section{B. Construction of the $\mathbf{O 2}$ bank}

The construction of a template bank relies on a number of parameters, including the selection of a representative noise power spectral density $S_{n}(f)$ and appropriate waveform models, the waveform starting frequency $f_{\text {low }}$, the placement method, and a specified minimum fitting factor criteria [48-50] for all templates in the bank.

The minimum fitting factor describes the effectualness of a template bank in recovering astrophysical sources. To define this quantity, we note that the matched filter output is maximized when a template waveform exactly overlaps the signal waveform. This optimization is impossible in practice, however, since the template bank samples the parameter space discretely while astrophysical sources arise from a continuum. Regardless, it is useful to quantify the degree to which two waveforms, $h_{1}$ and $h_{2}$, overlap. The overlap is defined as the noise-weighted inner product integral [48]:

$$
\left(h_{1} \mid h_{2}\right)=2 \int_{f_{\text {low }}}^{\infty} \frac{\tilde{h}_{1}(f) \tilde{h}_{2}^{*}(f)+\tilde{h}_{1}^{*}(f) \tilde{h}_{2}(f)}{S_{n}(f)} d f
$$

where $f_{\text {low }}$ was set to $15 \mathrm{~Hz}$, as motivated by the noise power spectral density described in Sec. II B 1.

The match between two waveforms is then defined as the noise-weighted inner product [48,49], maximized over a set of parameters denoted by $\phi$. For precessing signals, this overlap calculation considers only the $(2,2)$ mode and maximizes over the template's coalescence phase, polarization and sky position, while the overlap calculation for the higher order mode waveforms maximizes over only the polarization and sky position: 


$$
M\left(h_{1}, h_{2}\right)=\max _{\phi}\left(h_{1} \mid h_{2}(\phi)\right)
$$

This defines the percent of signal-to-noise ratio (SNR) retained when recovering waveform $h_{2}$ with the (nonidentical) waveform $h_{1}$. Then, the fitting factor is the related quantity used in describing the effectualness of template banks:

$$
F F\left(h_{s}\right)=\max _{h \in\left\{h_{b}\right\}} M\left(h_{s}, h\right),
$$

where $h_{b}$ is the set of templates in the bank and $h_{s}$ is a signal waveform with parameters drawn from the continuum. For the aligned spin waveforms, the $F F$ is calculated by maximizing the noise-weighted inner product only over the templates. The fitting factor describes the fraction of SNR retained for arbitrary signals in the parameter space covered by the bank. Typically, compact binary coalescence searches have required a fitting factor of $97 \%$ to ensure that no more than $\sim 10 \%$ of possible astrophysical signals are lost due to the discrete nature of the bank. As described in Sec. IIB 3, we use a hierarchical set of fitting factor requirements to construct the bank.

\section{Modeling the detector noise}

The noise power spectral density (PSD), as shown in Fig. 3, was used to compute the overlap integrals in the construction of the $\mathrm{O} 2$ template bank. This projected $\mathrm{O} 2$ sensitivity curve was produced by combining some of the best the LIGO Livingston detector (L1) sensitivities achieved before the start of the $\mathrm{O} 2$. At low frequencies, below $100 \mathrm{~Hz}$, the best sensitivity was taken from L1

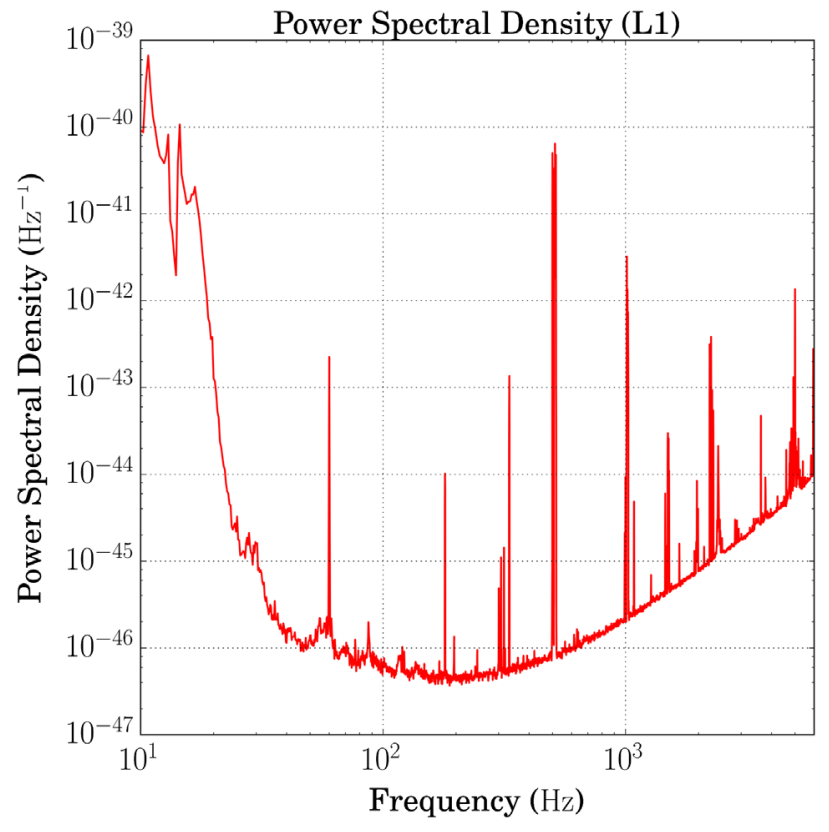

FIG. 3. Representation of the model power spectral density of detector noise. This was used to construct the $\mathrm{O} 2$ template bank. measurements during commissioning in February 2016. At high frequencies, above $100 \mathrm{~Hz}$, the best sensitivity is taken from $\mathrm{L} 1$ during the $\mathrm{O} 1$, with projected improved shot noise due to slightly higher input power and improved efficiency of the readout chain. Calculation of this PSD has been documented in [51].

\section{Waveform approximants}

Gravitational waveforms from compact binary mergers are described by 17 intrinsic and extrinsic parameters. However, as demonstrated in [52], for template placement purposes, we can parametrize these systems by three parameters composed of component masses $m_{i}$ and a reduced-spin parameter, as defined in [52], comprising the nonprecessing spin of the waveform, which is a function of the dimensionless spin parameters $\chi_{i}$ for $i=1,2$.

Above a total mass of $4 \mathrm{M}_{\odot}$, the waveforms of the binary systems are computed using the effective-one-body formalism (SEOBNRv4_ROM) [53], combining results from the post-Newtonian approach, black hole perturbation theory and numerical relativity to model the complete inspiral, merger and ringdown waveform. For binaries with total mass $\leq 4 \mathrm{M}_{\odot}$, waveforms are approximated by postNewtonian inspiral templates accurate to third-and-a-half order (the TaylorF2 approximant) [26,54]. The extent of the present parameter space covered by the template bank is limited by the availability of waveform models and the sensitivity of the present search. We neglect the effect of precession and higher order modes in our templates.

\section{Template placement}

Both the $\mathrm{O} 2$ off-line and online template banks were created in the same way, by constructing two sub-banks that were added together. For systems with total mass $2 \mathrm{M}_{\odot} \leq M \leq 4 \mathrm{M}_{\odot}$, where the TaylorF2 approximant is used, the templates were first laid out using a geometric metric technique [55]. This geometric bank was used as a coarse seed bank for an additional stochastic method placement [52,56], where templates were laid down densely enough to allow only a 3\% loss in SNR from a template not exactly matching a gravitational-wave signal, thus satisfying a minimum match set to $97 \%$. For systems with total mass greater than $4 \mathrm{M}_{\odot}$, where the SEOBNRv4_ROM approximant is used, a coarse bank was first generated with the stochastic method but with a very low minimum match. Again this stochastic bank was used as a coarse seed bank for an additional stochastic method placement with a minimum match set to $97 \%$. Additionally, only waveforms with a duration longer than a threshold of $0.2 \mathrm{~s}$, chosen in an ad hoc manner, were retained initially to avoid recovering short transient noise glitches, which are mostly seen to ring up heavier mass templates with shorter duration. The two sub-banks were added to form the full bank with a total of 661,335 templates. 


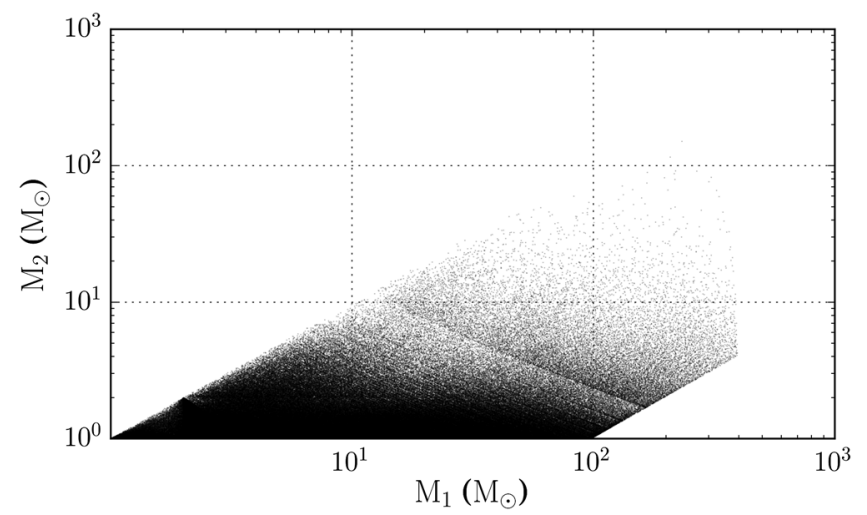

FIG. 4. A visual representation of the original $\mathrm{O} 2$ bank in the component mass space, containing a total of 661,335 templates placed with a minimal match of $97 \%$.

The original $\mathrm{O} 2$ off-line bank, as shown in Fig. 4, aided in the discovery of one of the earliest events detected during the O2, GW170104 [6]. The higher density of the bank at lower masses is expected because low mass systems have longer waveforms and spend more time in the detectors' sensitive frequency band. This enables the matched-filter search to better distinguish between two different low mass systems. This also means that more templates are required in the lower mass region of the bank for the required minimum match. At the highest masses, the waveforms contain very few cycles and very few templates are required for coverage in this region.

Early in the O2, short duration glitches were found to be particularly problematic for the online search, even with a duration cut of $0.2 \mathrm{~s}$ applied. Thus, to avoid delays in delivering low-latency gravitational-wave triggers, only waveforms with a total mass $<150 \mathrm{M}_{\odot}$ were retained in the online bank, based on our observations of the heavier mass templates being the most likely to recover noise triggers. This online bank, as shown in Fig. 2, was used for the entirety of the $\mathrm{O} 2$.

\section{Additional coverage in the off-line bank}

As outlined in Sec. II C, templates are grouped into bins by the GstLAL search such that all of the templates in any given bin have similar responses to noise, i.e., the distribution of SNR and $\chi^{2}$ in noise are similar for all of the templates in each bin [11]. It was uncovered partway through the $\mathrm{O} 2$ that the lower density of templates in the high mass part of the off-line bank (total mass $>80 \mathrm{M}_{\odot}$ ) was resulting in templates with very different background noise properties to be grouped together. This led to incorrect averaging of noise properties in the high mass groupings of templates and, in turn, resulted in incorrect estimation of the significance of loud coincident noise in time-shifted data from the two detectors [13]. This was not an issue in the online bank due to the cut at total mass $>150 \mathrm{M}_{\odot}$.

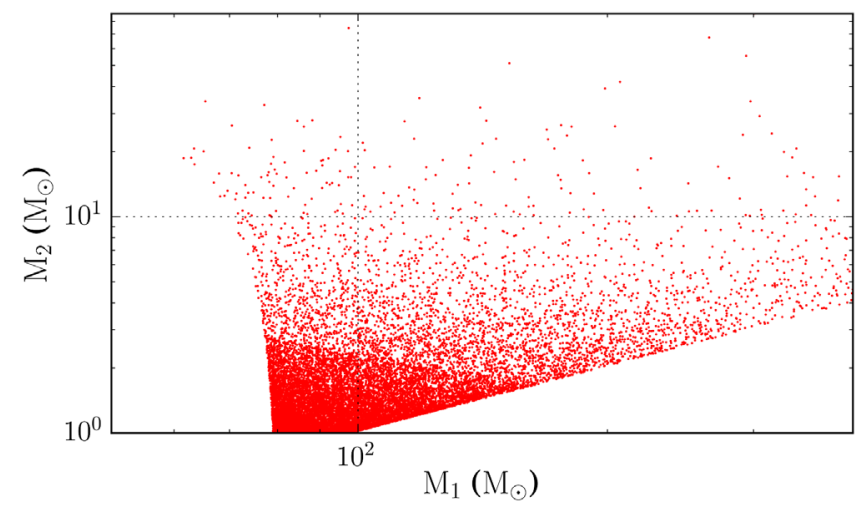

FIG. 5. The bank of the extra 14,665 templates that were added to the initial $\mathrm{O} 2$ bank, with a $98 \%$ minimum match above a total mass of $80 \mathrm{M}_{\odot}$ in the component mass space.

Two different recourses were taken. The off-line bank was overpopulated with extra templates in the higher mass region as outlined below. Additionally, the templates in this part of the bank were grouped differently from those in the denser lower mass region. Both of these steps were meant to ensure that more templates with a similar response to background noise can be grouped together, leading to a better estimation of the background noise captured by these templates. The bank was seen to reach its optimal sensitivity when these changes were applied to the higher mass region $80 \mathrm{M}_{\odot} \leq M \leq 400 \mathrm{M}_{\odot}$. Details of the template grouping methods are given in Ref. [13].

Regarding the additional coverage, extra templates were added to the initial off-line bank in the total mass range of $80 \mathrm{M}_{\odot} \leq M \leq 400 \mathrm{M}_{\odot}$ using two methods. As a first step, the original off-line bank was used as a seed for an additional stochastic placement in the total mass range $80 \mathrm{M}_{\odot} \leq M \leq 400 \mathrm{M}_{\odot}$ with an increased minimum match of $98 \%$. Initially, the template duration threshold of $0.2 \mathrm{~s}$ was chosen in an ad hoc manner in the hope that excluding these short duration templates would reduce the recovery of similar short duration glitches by them.

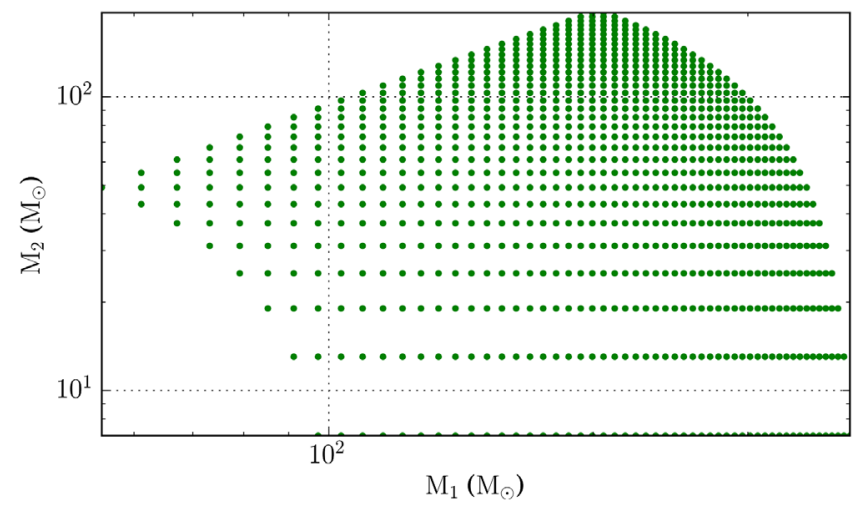

FIG. 6. The uniform grid bank with 1000 templates spanning $100-400 \mathrm{M}_{\odot}$ in total mass. 
TABLE I. Description of different categories of astrophysical populations, from which random mass and spin parameters were drawn and used to generate waveforms to check the effectualness of the template bank. Multiple simulation sets of the same population were used, varying in the type of waveform, mass ranges covered, and whether the spin is aligned to the orbital angular momentum.

\begin{tabular}{llll}
\hline \hline Population & Mass $\left(\mathrm{M}_{\odot}\right)$ & \multicolumn{1}{c}{ Spin } & \multicolumn{1}{c}{ Waveform approximant } \\
\hline BNS & $m_{1,2} \in[1,3]$ & $\chi_{1,2} \in[-0.05,0.05]$, aligned & TaylorF2 [54] \\
BNS & $m_{1,2} \in[1,3]$ & $\chi_{1,2} \in[-0.4,0.4]$, precessing & SpinTaylorT4 [58] \\
NSBH & $m_{2} \in[1,3]$ & $\chi_{1} \in[-0.4,0.4]$, aligned & SEOBNRv4_ROM [53] \\
& $m_{1} \in[3,97]$ & $\chi_{2} \in[-0.989,0.989]$, aligned & \\
NSBH & $m_{1} \in[3,15]$ & $\chi_{1} \in[-0.9,0.9]$, precessing & IMRPhenomPv2 [59] \\
& $m_{2} \in[1,3]$ & $\chi_{2} \in[-0.05,0.05]$, precessing & \\
BBH & $m_{1,2} \in[2,99]$ & $\chi_{1,2} \in[-0.99,0.99]$ aligned & SEOBNRv4_ROM [53] \\
BBH & $m_{1,2} \in[2,99]$ & $\chi_{1,2} \in[-0.99,0.99]$ precessing & SEOBNRv2_ROM_DoubleSpin [60] \\
IMBHB & $m_{1,2} \in[1,399]$ & $\chi_{1,2} \in[-0.998,0.998]$ aligned & SEOBNRv4_ROM [53] \\
IMBHB & $m_{1,2} \in[50,350]$ & Nonspinning & EOBNRv2HM [57] \\
\hline \hline
\end{tabular}
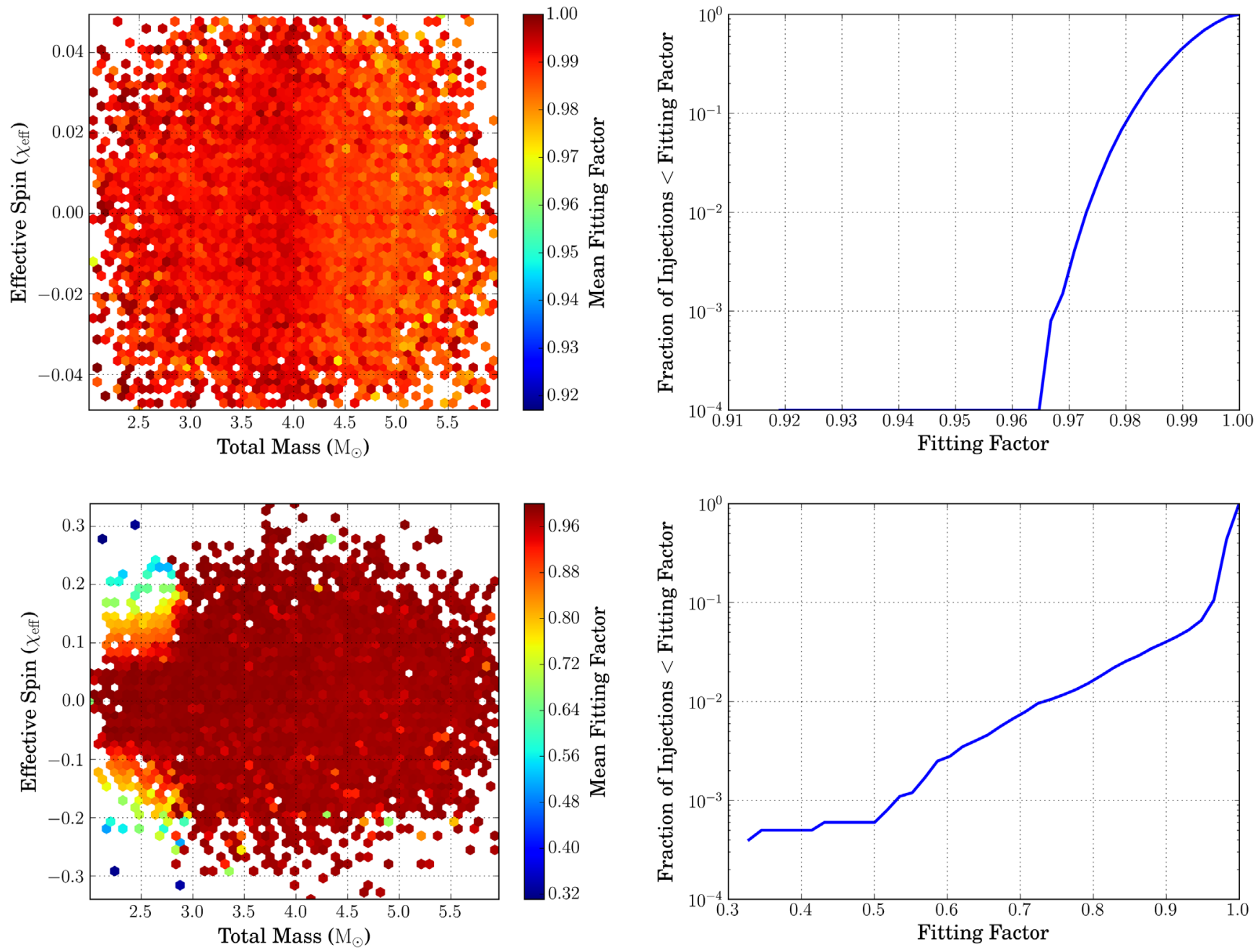

FIG. 7. Fitting factors in $M-\chi_{\text {eff }}$ plane for BNS aligned-spin TaylorF2 waveform approximants [54] (top left) and precessing-spin SpinTaylorT4 waveform approximants [58] (bottom left). We also include cumulative histograms of the fitting factors of the respective waveforms (top and bottom right). For the aligned-spin BNS systems, we recover $99.7 \%$ of the injected simulations with fitting factors above 0.97 . Hence, the majority of fitting factors are above 0.97 , except along the low-mass edge of the bank at $M=2.0$, where the fitting factor starts to fall off. The bank is constructed with TaylorF2 waveforms so fitting factors are expected to be at least as high as the required fitting factor of 0.97 to ensure that no more than $\sim 10 \%$ of possible astrophysical signals are lost due to the discrete nature of the bank. For the precessing BNS systems, we recover $86.1 \%$ of the injected simulations with fitting factors above 0.97 , although sensitivity falls off rapidly outside $-0.05<\chi_{\text {eff }}<0.05$ for systems with NS component mass less than $2 \mathrm{M}_{\odot}$. There are no templates placed in this region so the fall off in fitting factor is expected. This also demonstrates that a search based on an aligned-spin template bank can recover precessing-spin signals. 
We found this was not the case. Hence, additionally, no template duration threshold was used in these extra templates that were added to the mass range $80 \mathrm{M}_{\odot} \leq M \leq 400 \mathrm{M}_{\odot}$ so as to not exclude the short waveforms corresponding to the heavier mass systems. In the region $M \leq 80 \mathrm{M}_{\odot}$, the duration of the templates being much longer than $0.2 \mathrm{~s}$, they remained unaffected by this duration threshold. A total of 14,665 templates, as shown in Fig. 5, were added to the initial off-line bank.

Despite the increased minimum match, the high mass region of the bank remained sparsely populated, as the overlap between high mass waveforms with few cycles are generally high. Thus, the minimum match required is already met, without the placement of additional templates.
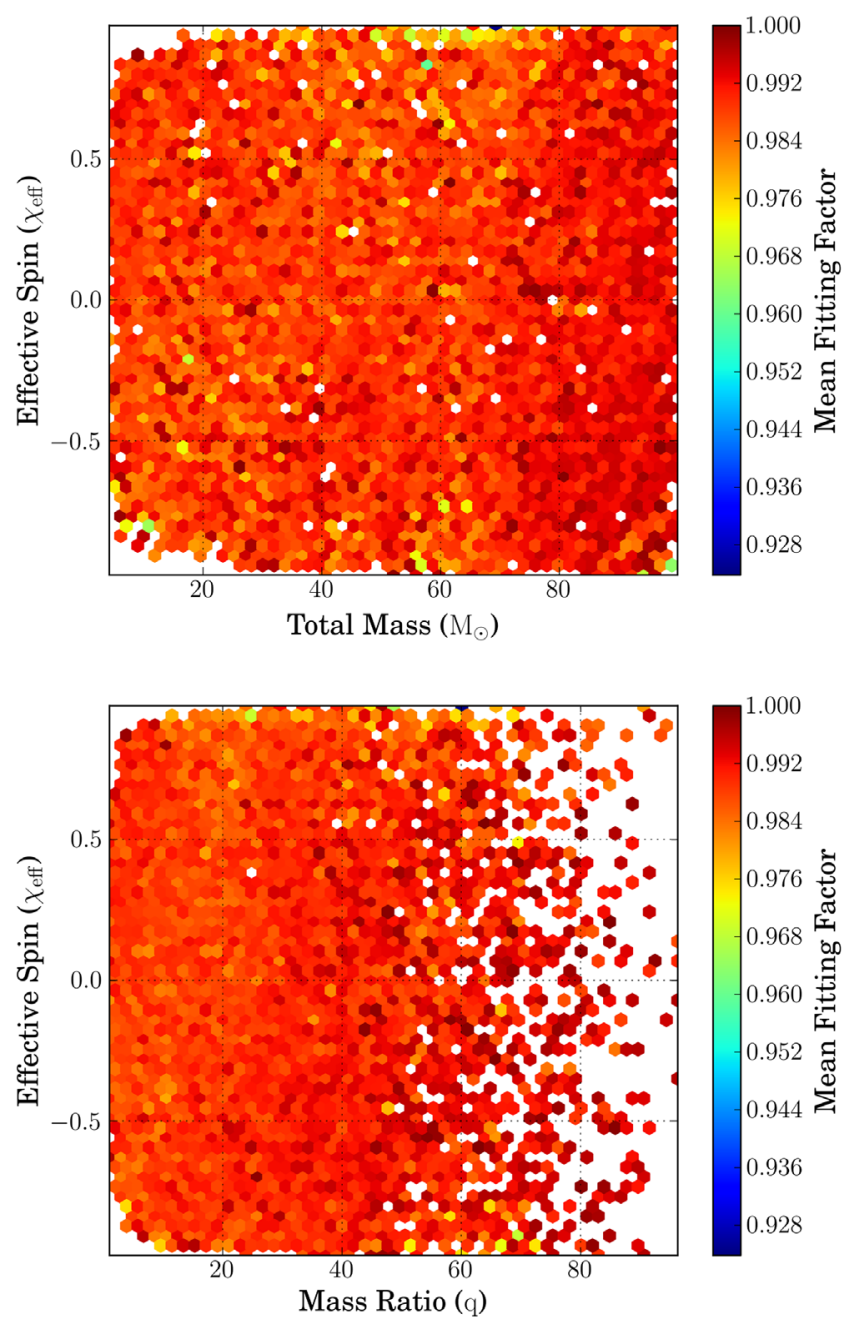

However, short duration glitches are also recovered by a relatively few number of high mass templates, and if these few glitchy templates are grouped together for background estimation with quieter templates, they can spoil the sensitivity over a broad mass range. Thus, we chose to force the placement of additional templates at higher mass using a uniform grid placement in component mass space for the total mass range between $100 \mathrm{M}_{\odot} \leq M \leq 400 \mathrm{M}_{\odot}$, with mass ratios between 1 and 97.988. A total of 1000 templates were placed without any limitations on the waveform duration. This gridded bank, as shown in Fig. 6, was then added to the off-line bank produced in the previous step.

All together, the final improved $\mathrm{O} 2$ bank has a total of about 677,000 templates, as shown in Fig. 1.
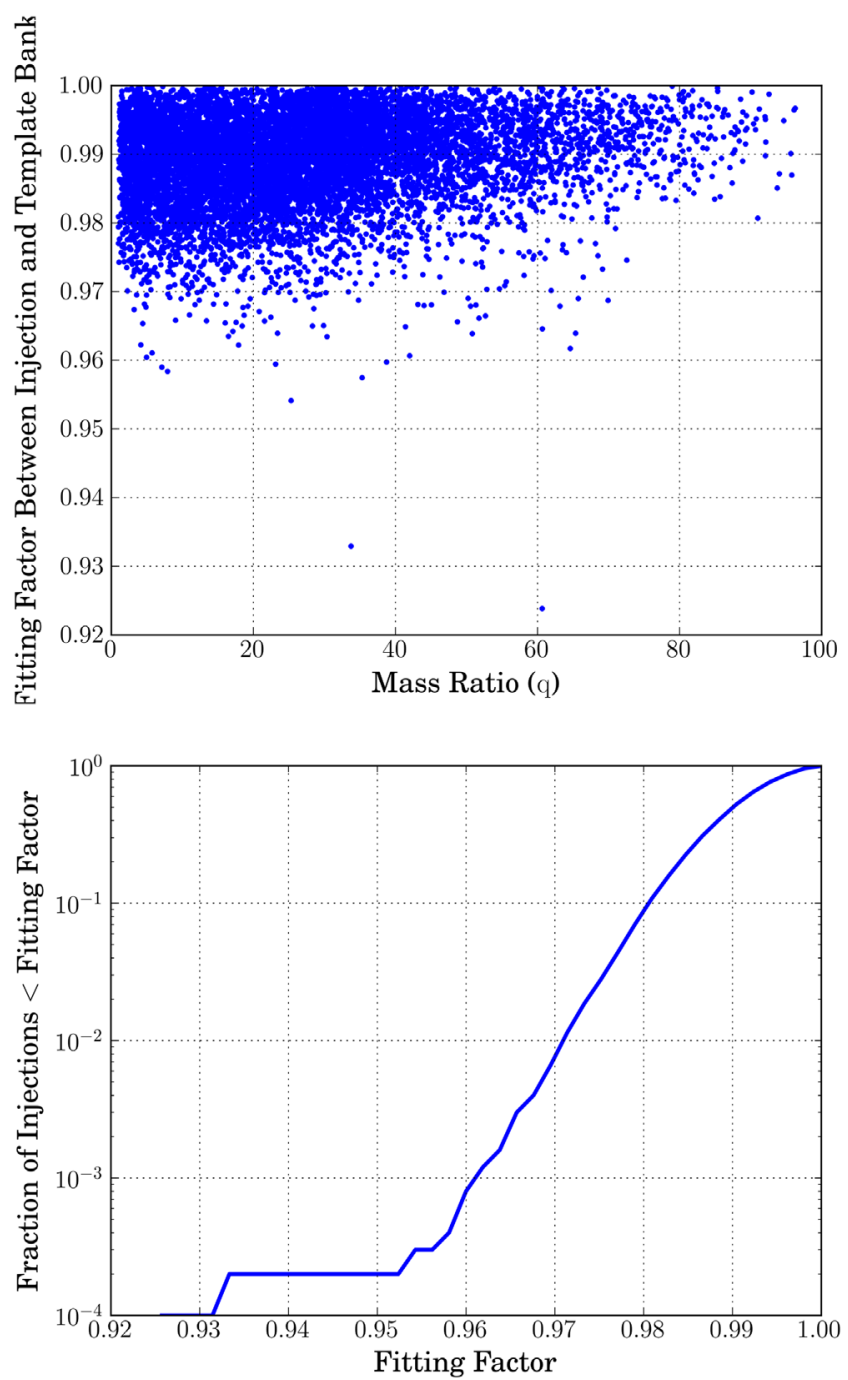

FIG. 8. Fitting factors in $M-\chi_{\text {eff }}$ plane (top left), as a function of mass ratio (q) (top right), and in the $q-\chi_{\text {eff }}$ plane (bottom left) for NSBH aligned-spin SEOBNRv4_ROM waveform approximants [53]. We also include a cumulative histogram of the fitting factors (bottom right). The majority $(99.2 \%)$ of fitting factors are above 0.97 . Lower fitting factors are expected for the remaining systems, where the neutron star component spin is higher than 0.05 or lower than -0.05 , as the NSBH part of the bank does not have templates here. However, the fit improves slightly at very high mass ratios (above about 70) since these systems are also high total mass systems, which are recovered by the part of the bank where additional templates were placed with a higher $(98 \%)$ minimum match. 


\section{Implementation in the GstLAL pipeline}

The GstLAL-based inspiral search is a matched-filtering pipeline. The noise-weighted inner product of each whitened template with the whitened data produces the SNR. Both signals and glitches can produce high SNR, thus, a number of additional consistency and coincidence checks are implemented in the pipeline, as detailed in Ref. [13]. In order to access the full waveform of binary systems up to $400 \mathrm{M}_{\odot}$ that merge at lower frequencies, the filtering frequency was reduced from $30 \mathrm{~Hz}$ in the $\mathrm{O} 1$ search to $15 \mathrm{~Hz}$.

In the GstLAL pipeline, the background characteristics are estimated for each detector independently using
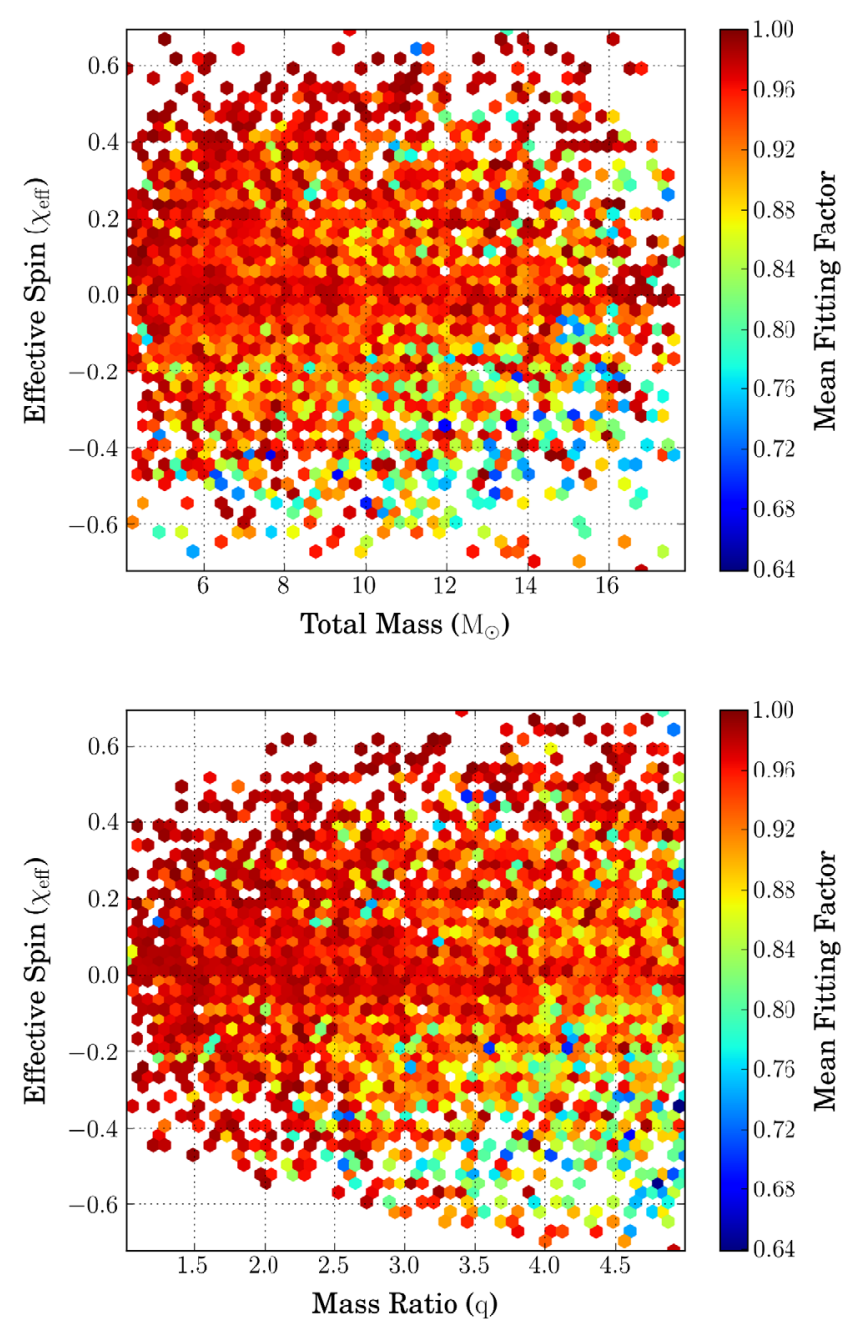

noncoincident triggers that occurred during times they could have formed a coincidence, i.e., noncoincident triggers that occurred when more than one detector had science quality data. This is done for several different bins of templates across the template bank, thus, each bin collects its own background statistics used for assigning likelihood ratios to candidates in that bin. In the region of the template bank, where the total mass is less than $80 \mathrm{M}_{\odot}$, the templates are binned first in the effective spin and then in chirp mass (as defined below); in the high mass region, templates are binned by template duration. These two techniques were experimentally found to group templates together that have similar responses to noise. More information on the spin and chirp mass binning can be found in
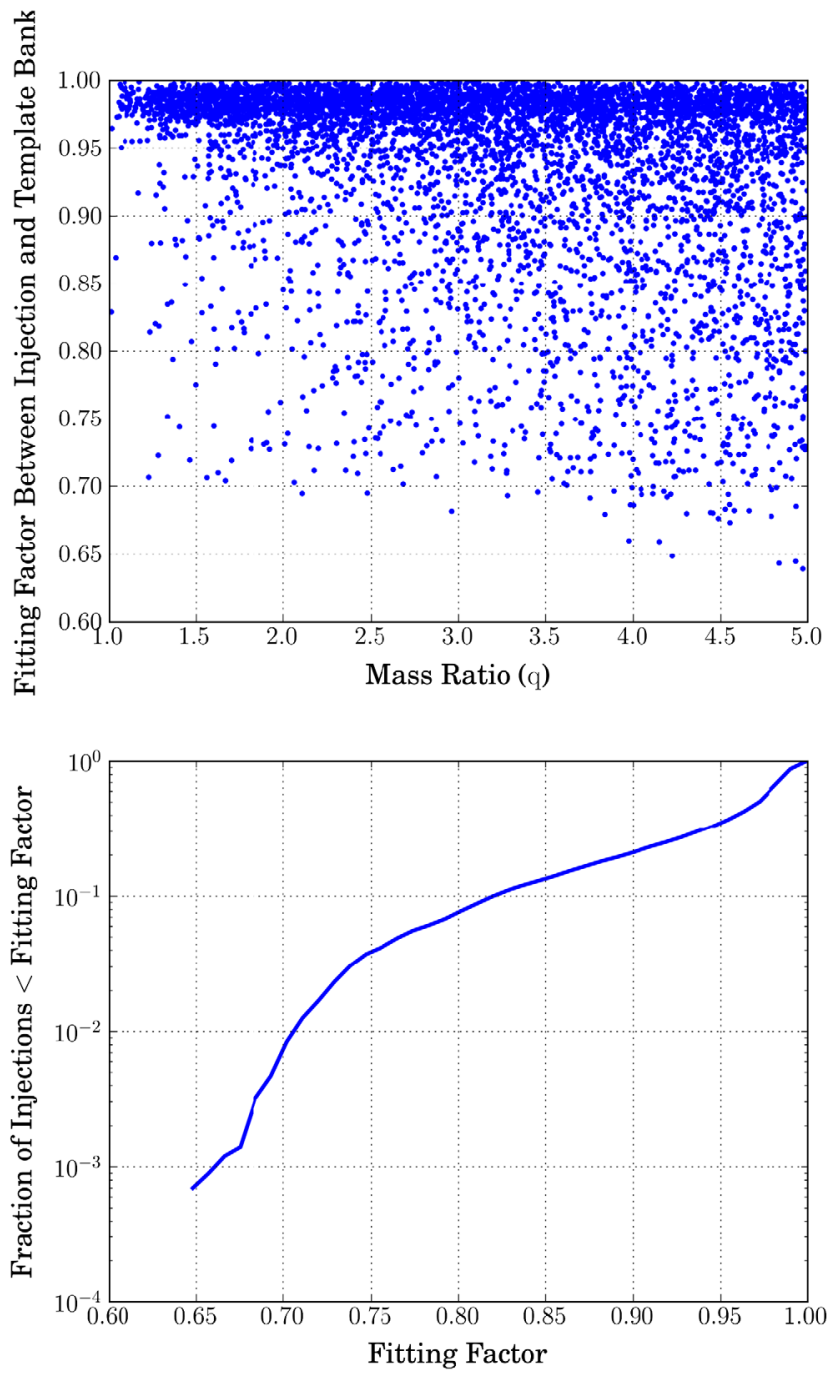

FIG. 9. Fitting factors in $M-\chi_{\text {eff }}$ plane (top left), as a function of mass ratio (q) (top right), and in the $q-\chi$ eff plane (bottom left) for NSBH precessing IMRPhenomPv2 waveform approximants [59]. We also include a cumulative histogram of the fitting factors (bottom right). Only $51.5 \%$ of fitting factors are above 0.97 and fitting factors down to below 0.65 are present across this region. Top right: we can see that the low fitting factors occur for systems with more extreme mass ratios, while (top left) tells us the fitting factors are lower for the highly spinning systems. This is what we expect since our bank does not have templates that include precession effects, which depend on the mass ratio and the effective spin of the binary. The combined effect can be seen in the (bottom left) plot, where the increased mass ratio and $\chi_{\text {eff }}$ shows lower fit. 
Ref. [11], and more information on the duration binning can be found in Ref. [13]. For the purpose of background estimation, templates are grouped together so that each group has templates with a similar response to background noise. Noise properties are averaged separately for each group. Before the $\mathrm{O} 2$, templates were grouped according to two composite parameters that characterize the waveform inspiral to leading order. As also pointed out in [11,13], these were the chirp mass of the binary $\mathcal{M}$ and the effective spin parameter $\chi_{\text {eff. }}$ The chirp mass is

$$
\mathcal{M}=\frac{\left(m_{1} m_{2}\right)^{3 / 5}}{\left(m_{1}+m_{2}\right)^{1 / 5}}
$$

The effective spin parameter is defined as

$$
\chi_{\text {eff }} \equiv \frac{m_{1} \chi_{1}+m_{2} \chi_{2}}{m_{1}+m_{2}}
$$
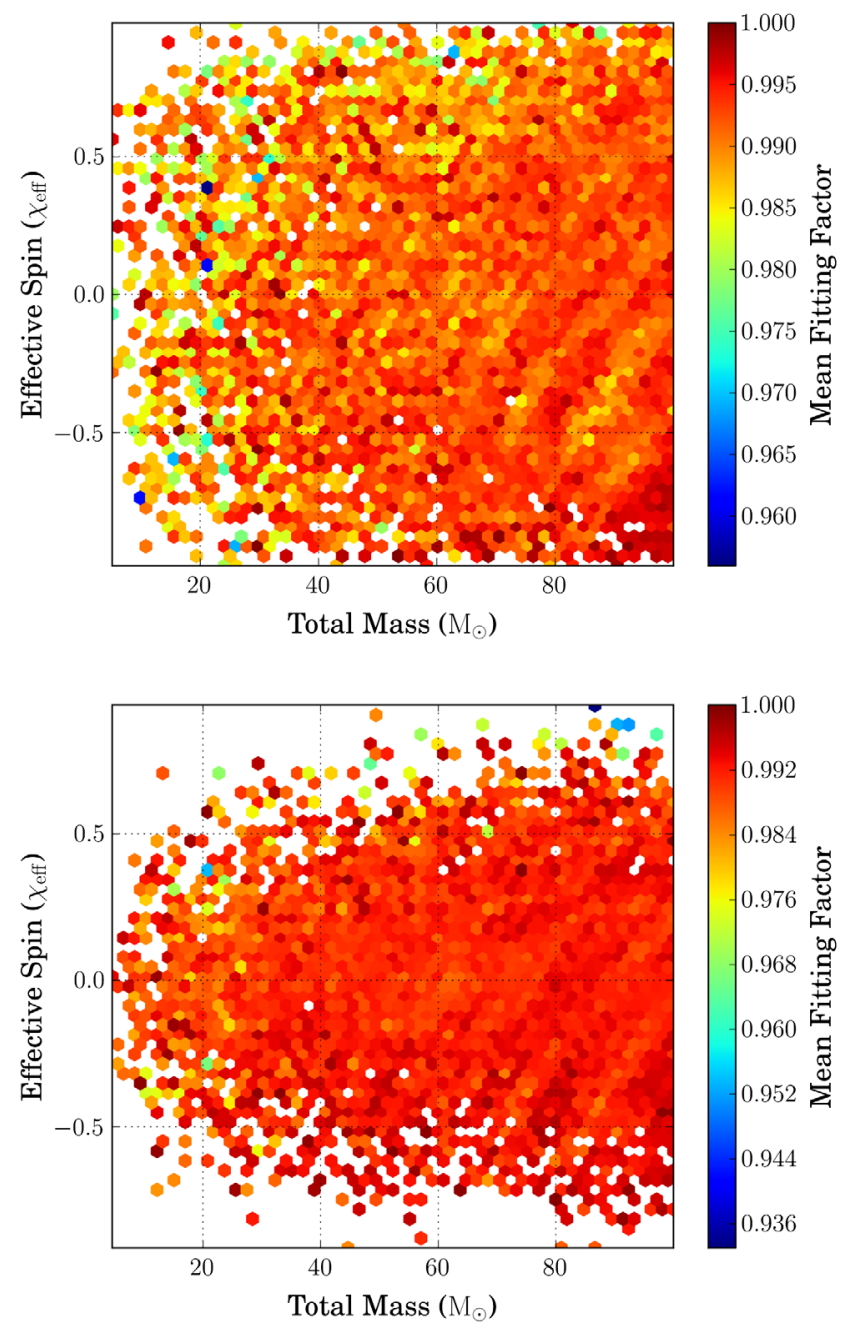

and acts as a mass-weighted combination of the spin components (anti-)aligned with the total angular momentum.

However, as described in Sec. II B 4, extra templates were placed in the high mass region of the off-line bank to better capture the properties of the noise in that regime. Templates above a total mass of $80 \mathrm{M}_{\odot}$ were then grouped by template duration from $15 \mathrm{~Hz}$ rather than the $\mathcal{M}$ and $\chi_{\text {eff }}$ binning used at lower masses. Template duration better characterizes the waveform merger and ringdown, the detectable part of the signal for high mass systems.

\section{EFFECTUALNESS}

To assess the effectualness of this template bank, we compute again the $F F\left(h_{s}\right)$, as defined in Eq. (3), for a collection of simulated signals with parameters drawn randomly from the covered mass and spin space. The $F F$ depends on the parameters of the simulations and varies
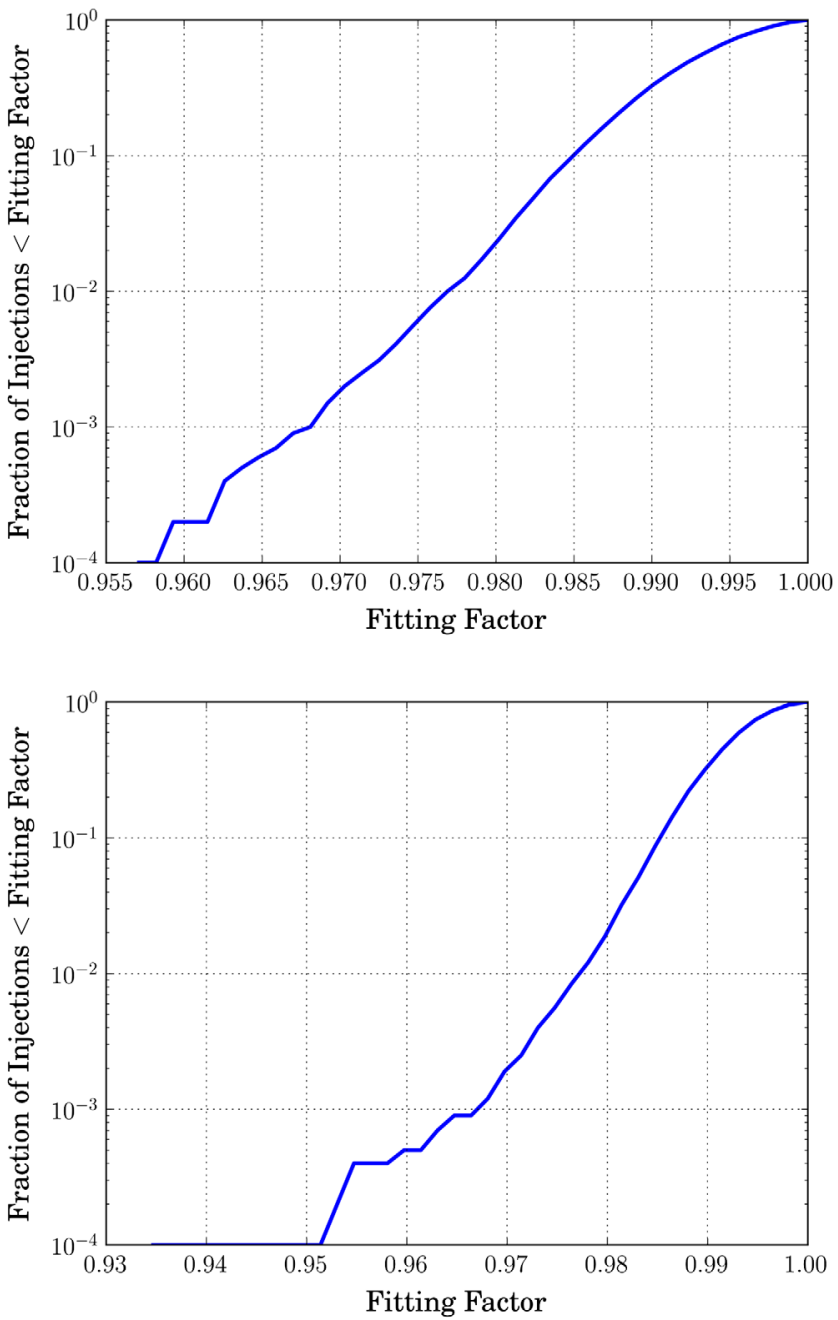

FIG. 10. Fitting factors in $M-\chi_{\text {eff }}$ plane for BBH aligned-spin SEOBNRv4_ROM waveform approximants [53] (top left) and precessing-spin SEOBNRv2_ROM_DoubleSpin waveform approximants [60] (bottom left). We also include cumulative histograms of the fitting factors of the respective waveforms (top and bottom right). For the aligned-spin BBH systems, we recover $99.8 \%$, and for the precessing $\mathrm{BBH}$ systems, we recover $99.79 \%$ of the injected simulations with fitting factors above 0.97 . 
across the parameter space of the bank, hence, it can be represented and plotted as a function of two of the parameters of the simulations. In order to do so, the $F F$ is binned in the two parameters and the mean $F F$ in each bin is plotted [49]. We show such plots in parameters like chirp masses, mass ratios, and effective spins:

$$
F F_{\text {mean }}=\langle F F\rangle \text {. }
$$

We selected simulated signals from various populations of BNS, NSBH, BBH, and IMBHB systems to check the effectualness of the bank. The details of the simulation sets are summarized in Table I. The simulated signals were chosen to be uniformly distributed on the sky and placed at a fixed luminosity distance of $1 \mathrm{Mpc}$. Precessing and higher-order mode signals were given binary inclination angles $l$ uniformly distributed in $\arccos t$. The nonspinning IMBHB population, described by the EOBNRv2HM [57] waveforms, was also selected to be distributed uniformly in total mass. For the aligned spin binary systems, the extrinsic parameters are not explicitly used in the calculation of effectuality, and the waveforms can be generated using the same fiducial extrinsic parameters and are normalized so that the fitting factor of a waveform that has the same parameters as one of the templates is 1 . For the precessing simulations, the $F F$ calculation maximizes over coalescence phase, polarization, and sky position, and for those including higher order mode contributions, it maximizes over only the polarization and sky position. For each signal population, $10^{4}$ simulations were performed.

In Fig. 7, we can see the fitting factors in the $M-\chi_{\text {eff }}$ plane for BNS aligned-spin TaylorF2 waveform approximants [54]
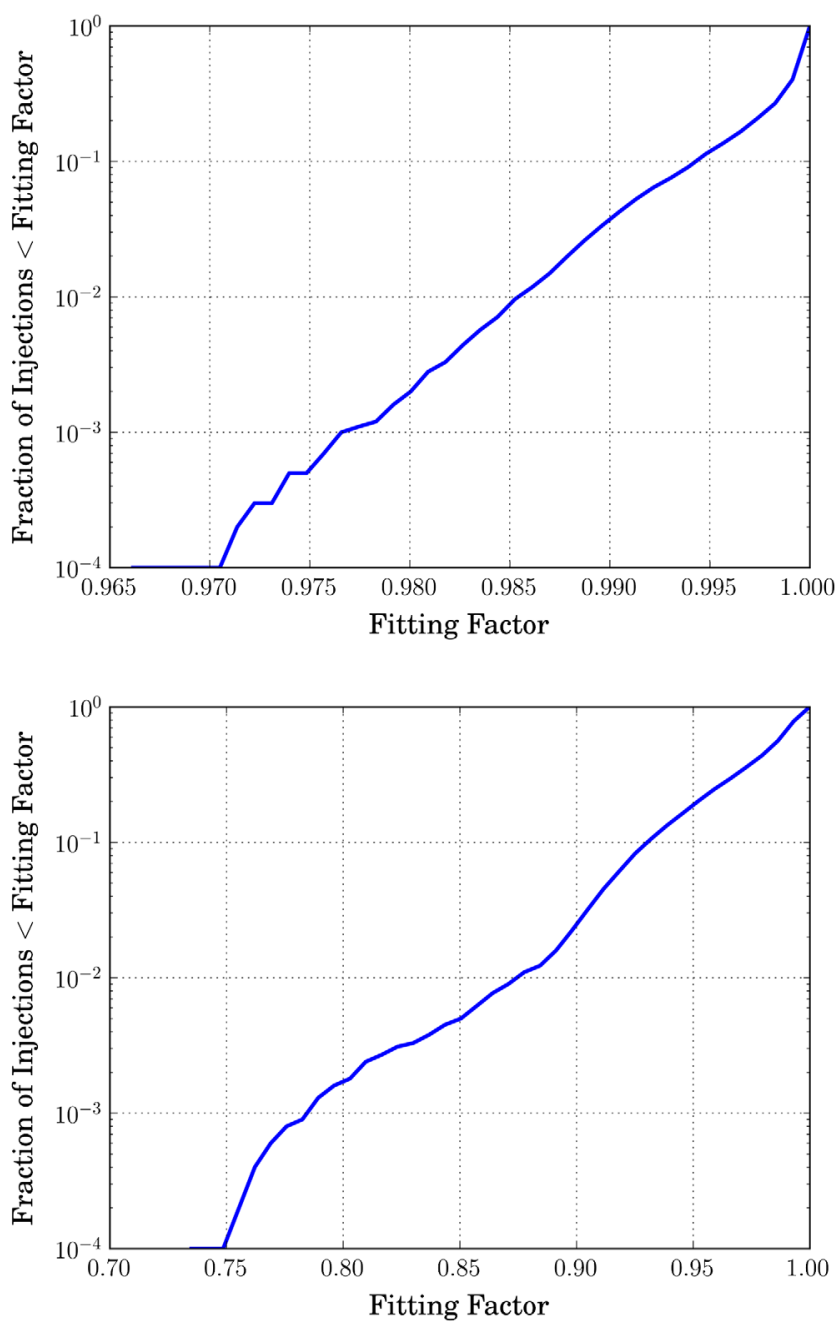

FIG. 11. Fitting factors in $M-\chi$ eff plane for IMBHB aligned-spin SEOBNRv4_ROM waveform approximants [53] (top left) and as a function of mass ratio for IMBHB nonspinning EOBNRv2HM waveform approximants [57] (bottom left). Cumulative histograms of the fitting factors of the respective waveforms is also included (top and bottom right). $99.99 \%$ of fitting factors are above 0.97 for the recovery of aligned-spin SEOBNRv4_ROM waveform approximants. Nonspinning EOBNRv2HM waveform approximants with higher-order modes can also be recovered by the search in the IMBHB region, despite template waveforms not including higher-order mode effects. We recover $66.6 \%$ of these simulations with fitting factors above 0.97 . 
and precessing-spin SpinTaylorT4 waveform approximants [58]. We also include cumulative histograms of the fitting factors of the respective waveforms. 99.7\% of aligned-spin BNS simulations were recovered with a fitting factor above 0.97 , while $86.1 \%$ of the precessing BNS simulations were recovered with fitting factors above 0.97 . Hence, for the aligned-spin BNS systems, the majority of fitting factors are above 0.97, except along the low-mass edge of the bank at $M=2.0 \mathrm{M}_{\odot}$ below which no templates are placed. The bank is constructed with aligned-spin TaylorF2 waveforms in this low mass region so fitting factors are expected to be at least as high as the required fitting factor of 0.97 to ensure that no more than $\sim 10 \%$ of possible astrophysical signals are lost due to the discrete nature of the bank. For the precessing-spin SpinTaylorT4 waveform approximants the fit falls off rapidly outside $-0.05<\chi_{\text {eff }}<0.05$ for systems with NS component mass less than $2 \mathrm{M}_{\odot}$. There are no templates placed in this region so the fall off in fitting factor is expected. This also demonstrates that a search based on an aligned-spin template bank can recover precessing-spin signals.

In Fig. 8, we can see the fitting factors in the $M-\chi_{\text {eff }}$ plane and as a function of mass ratio for NSBH aligned-spin SEOBNRv4_ROM waveform approximants [53]. For 99.2\% of the simulations, the fitting factors are above 0.97. Lower fitting factors are expected for the remaining binary systems where the neutron star component spin is higher than 0.05 or lower than -0.05 , as the NSBH part of the bank does not have templates here. However, the fit improves slightly at very high mass ratios (above about 70) since these systems are also high total mass systems, which are recovered by the part of the bank where additional templates were placed with a higher $(98 \%)$ minimum match.

The precessing NSBH simulations using the IMRPhenomPv2 waveform [59] in Fig. 9 seem to be recovered with a lower fit for the highly precessing systems with high effective spins and mass ratios, and only 51.5\% of the fitting factors are above 0.97 . This is what we expect since our bank does not have templates that include precession effects, which depend on the mass ratio and the effective spin of the binary.

In Figs. 10 and 11, we can see the fitting factors in $M-\chi_{\text {eff }}$ plane for $\mathrm{BBH}$ and IMBHB aligned-spin SEOBNRv4_ROM waveform approximants [53], the precessing BBH SEOBNRv2_ROM_DoubleSpin waveform approximants [60], and as a function of mass ratio for IMBHB nonspinning EOBNRv2HM waveform approximants [57]. For the aligned-spin SEOBNRv4_ROM waveform approximants, $99.8 \%$ of the injected $\mathrm{BBH}$ simulations and $99.99 \%$ of the IMBHB simulations are recovered with fitting factors above 0.97 . The bank is constructed with SEOBNRv4_ROM waveforms in the high mass region so fitting factors are expected to be at least as high as the required fitting factor of 0.97 . Even though the bank does not include precessing templates, $99.79 \%$ of the precessingspin SEOBNRv2_ROM_DoubleSpin BBH waveform approximants are recovered with fitting factors above 0.97. Nonspinning EOBNRv2HM waveform approximants, including modes higher than the fundamental $(2,2)$ mode, can also be recovered by the search in the IMBHB region, despite template waveforms not including such higher-order mode effects. We recover $66.6 \%$ of these simulations with fitting factors above 0.97 , and the fit is seen to decline at higher mass ratios. This is because the fitting factors have a dependency on mass ratios, as effects of higher-order modes become more significant at higher total mass and mass ratios [24].

\section{CONCLUSION}

We have presented the construction and effectualness of the aligned-spin online and off-line template banks of gravitational waveforms used by the GstLAL-based inspiral pipeline to analyze data from the second observing run of Advanced LIGO and Virgo. The off-line bank expands upon the parameter space covered during the first observing run, including coverage for merging compact binary systems with total mass between $2 \mathrm{M}_{\odot}$ and $400 \mathrm{M}_{\odot}$ and mass ratios between 1 and 97.988, thus expanding into the intermediate-mass binary black hole range. The bank requires that templates with component masses less than $2 \mathrm{M}_{\odot}$ have (anti-)aligned spins between \pm 0.05 , while component masses greater than $2 \mathrm{M}_{\odot}$ have allowed (anti-)aligned between \pm 0.999 . Despite this aligned-spin constraint, we find that the bank can recover some precessing-spin systems. Additionally, higher-order mode effects are not included in the template waveforms, but the bank can recover some nonspinning IMBHB waveforms with higher-order modes.

We expect to be sensitive to BNSs with component masses 1-3 $\mathrm{M}_{\odot}$ and (anti-)aligned spins between \pm 0.05 . Our sensitivity to precessing BNS systems with spins (anti-)aligned above 0.05 or below -0.05 is limited as discussed in Sec. III and in Sec. II A. We are sensitive to NSBH systems with component masses in the range 1-3 and 3-97 $\mathrm{M}_{\odot}$ and (anti-)aligned spins between \pm 0.05 for the neutron star component and between \pm 0.99 for the black hole component. Precessing NSBH systems and ones with component spins outside this range are recovered with poorer fits. We are most sensitive to BBH systems with component masses in the range $2-99 \mathrm{M}_{\odot}$ and (anti-)aligned spins in the range \pm 0.99 and have poorer recovery for precessing systems. We are the most sensitive to IMBHB systems with component masses between 1-399 $\mathrm{M}_{\odot}$, total mass in the range $100-400 \mathrm{M}_{\odot}$, and (anti-)aligned spins in the range \pm 0.998 . Recovery for IMBHB waveforms, including effects of higher order modes, is seen to fall with increase in mass ratios. Higher-order modes have higher frequency content and will be within the sensitive frequency band of LIGO and Virgo for IMBHB signals. Hence, it will become important to include templates containing higher-order mode 
contributions, in order to increase the sensitivity of the search towards heavier mass systems [24].

The online and off-line banks played key roles in the discoveries of the $\mathrm{O} 2[6-8,10]$ and the off-line bank was used in the deep GstLAL reanalysis of the $\mathrm{O} 1$ and $\mathrm{O} 2$ [9]. The present bank, however, contains templates that assume the spins of the stars to be aligned with their orbital angular momentum. They do not include precession. Hence, we see precessing waveforms are recovered with a lower fit with the templates that recover them. The bank also does not include templates with higher order modes in their waveforms, which has been seen to reduce our sensitivity to them. The bank includes templates for systems with mass ratios going from 1 to 97.988 , and it could be expanded to include contributions from higher order modes. From the construction of this template bank, we also learned that we need optimal coverage in the region representing the higher mass IMBHB systems to better estimate the background statistics. We additionally presented a new method that combines a stochastic method with a grid-bank method to better isolate noisy templates at the high mass region of the bank. This allowed for better grouping of templates when performing background estimation. A more careful layout and grouping of templates has been implemented in our template bank for the third observing run, which will be covered in a future work. The experience gained in designing these banks has informed the construction of the template bank, which is being used for the third observing run of Advanced LIGO and Virgo.

We use the LALApps package from the LIGO Algorithm Library [61] to generate the waveforms and to construct and validate the bank. The plots were made using Matplotlib [62].

\section{ACKNOWLEDGMENTS}

We thank the LIGO-Virgo Scientific Collaboration for access to data. LIGO was constructed by the California
Institute of Technology and Massachusetts Institute of Technology with funding from the National Science Foundation (NSF) and operates under cooperative agreement PHY-0757058. We thank Satya Mohapatra for the helpful comments and suggestions. We also thank Graham Woan for helping with the review of the template bank used for the O2. We gratefully acknowledge the support by NSF Grant No. PHY-1607585 for J. C., P. B., D. C. and D. M. D. M. was also partly supported by PHY 14-54389, ACI 16-42391, OAC 18-41480. S.C. is supported by the research program of the Netherlands Organisation for Scientific Research (NWO). S. S. was supported in part by the Eberly Research Funds of Penn State, The Pennsylvania State University, University Park, PA 16802, USA.H.F. was supported by the Natural Sciences and Engineering Research Council of Canada (NSERC). C. H. was supported in part by the NSF through Grant No. PHY-1454389. Funding for this project was provided by the Charles E. Kaufman Foundation of The Pittsburgh Foundation. T. G. F. L. was partially supported by a grant from the Research Grants Council of the Hong Kong (Project No. CUHK 14310816 and CUHK 24304317) and the Direct Grant for Research from the Research Committee of the Chinese University of Hong Kong. M. W. was supported by NSF Grant No. PHY1607178. We thank the computational resources provided by Leonard E. Parker Center for Gravitation, Cosmology and Astrophysics at the University of Wisconsin-Milwaukee and supported by National Science Foundation Grants No. PHY-1626190 and No. PHY-1700765. We are also grateful for the computational resources provided by The Pennsylvania State University's Institute for CyberScience Advanced CyberInfrastructure (ICS-ACI). The authors are also grateful for computational resources provided by the LIGO Laboratory and supported by National Science Foundation Grants No. PHY-0757058 and No. PHY-0823459.
[1] G. M. Harry et al. (LIGO Scientific Collaboration), Classical Quant. Grav. 27, 084006 (2010).

[2] J. Aasi et al. (LIGO Scientific Collaboration), Classical Quant. Grav. 32, 074001 (2015).

[3] B. P. Abbott et al. (LIGO Scientific and Virgo Collaborations), Phys. Rev. Lett. 116, 061102 (2016).

[4] A. Albert et al. (ANTARES, IceCube, LIGO Scientific, and Virgo Collaborations), Phys. Rev. D 96, 022005 (2017).

[5] F. Acernese, M. Agathos, K. Agatsuma et al., Classical Quant. Grav. 32, 024001 (2015).

[6] B. P. Abbott et al. (LIGO Scientific and Virgo Collaborations), Phys. Rev. Lett. 118, 221101 (2017).

[7] B. P. Abbott et al., Astrophys. J. Lett. 851, L35 (2017).
[8] B. P. Abbott et al. (LIGO Scientific and Virgo Collaborations), Phys. Rev. Lett. 119, 141101 (2017).

[9] B. P. Abbott et al. (LIGO Scientific and Virgo Collaborations), Phys. Rev. X 9, 031040 (2019).

[10] B. P. Abbott et al. (LIGO Scientific and Virgo Collaborations), Phys. Rev. Lett. 119, 161101 (2017).

[11] C. Messick, K. Blackburn, P. Brady et al., Phys. Rev. D 95, 042001 (2017).

[12] K. Cannon, C. Hanna et al., GstLAL, https://www.lsc-group .phys.uwm.edu/daswg/projects/gstlal.html.

[13] S. Sachdev et al., arXiv:1901.08580.

[14] K. Cannon et al., Astrophys. J. 748, 136 (2012).

[15] S. Privitera et al., Phys. Rev. D 89, 024003 (2014). 
[16] C. Hanna et al., Phys. Rev. D 101, 022003 (2020).

[17] T. Dal Canton, A. H. Nitz, A. P. Lundgren et al., Phys. Rev. D 90, 082004 (2014).

[18] S. Usman, A. H. Nitz, I. W. Harry et al., Classical Quant. Grav. 33, 215004 (2016).

[19] A. H. Nitz et al., gwastro/pycbc: 1.18 .0 release of PyCBC, Zenodo, https://doi.org/10.5281/zenodo.596388 (2017).

[20] A. H. Nitz, Classical Quant. Grav. 35, 035016 (2018).

[21] T. Adams, D. Buskulic, V. Germain, G. M. Guidi, F. Marion, M. Montani, B. Mours, F. Piergiovanni, and G. Wang, Classical Quant. Grav. 33, 175012 (2016).

[22] S. Klimenko, I. Yakushin, A. Mercer, and G. Mitselmakher, Classical Quant. Grav. 25, 114029 (2008).

[23] B. P. Abbott, R. Abbott, T. D. Abbott et al., Phys. Rev. D 100, 064064 (2019).

[24] J. Calderón Bustillo, F. Salemi, T. Dal Canton, and K. P. Jani, Phys. Rev. D 97, 024016 (2018).

[25] P. Kumar, I. MacDonald, D. Brown et al., Phys. Rev. D 89, 042002 (2014).

[26] B. P. Abbott et al. (LIGO Scientific and Virgo Collaborations), Phys. Rev. D 93, 122003 (2016).

[27] B. P. Abbott et al. (LIGO Scientific and Virgo Collaborations), Phys. Rev. X 6, 041015 (2016).

[28] T. Dal Canton and I. W. Harry, arXiv:1705.01845.

[29] B. P. Abbott et al. (LIGO Scientific and Virgo Collaborations), Phys. Rev. D 96, 022001 (2017).

[30] C. E. Rhoades and R. Ruffini, Phys. Rev. Lett. 32, 324 (1974).

[31] V. Kalogera and G. Baym, Astrophys. J. Lett. 470, L61 (1996).

[32] F. Özel, D. Psaltis, R. Narayan, and A. S. Villarreal, Astrophys. J. 757, 55 (2012).

[33] J. M. Lattimer, Annu. Rev. Nucl. Part. Sci. 62, 485 (2012).

[34] B. Kiziltan, A. Kottas, M. D. Yoreo, and S. E. Thorsett, Astrophys. J. 778, 66 (2013).

[35] M. Linares, T. Shahbaz, and J. Casares, Astrophys. J. 859, 54 (2018).

[36] F. Özel and P. Freire, Annu. Rev. Astron. Astrophys. 54, 401 (2016).

[37] R. O’Shaughnessy, J. Kaplan, V. Kalogera, and K. Belczynski, Astrophys. J. 632, 1035 (2005).

[38] K. Belczynski, A. Buonanno, M. Cantiello, C. L. Fryer, D. E. Holz, I. Mandel, M. C. Miller, and M. Walczak, Astrophys. J. 789, 120 (2014).

[39] S. E. de Mink and K. Belczynski, Astrophys. J. 814, 58 (2015).

[40] M. C. Miller and E. J. M. Colbert, Int. J. Mod. Phys. D 13, 1 (2004).

[41] B. P. Abbott et al. (Virgo and LIGO Scientific Collaborations), Phys. Rev. Lett. 121, 231103 (2018).
[42] J. W. T. Hessels, S. M. Ransom, I. H. Stairs, P. C. C. Freire, V. M. Kaspi, and F. Camilo, Science 311, 1901 (2006).

[43] M. Kramer and N. Wex, Classical Quant. Grav. 26, 073001 (2009).

[44] A. C. Fabian, D. R. Wilkins, J. M. Miller, R. C. Reis, C. S. Reynolds, E. M. Cackett, M. A. Nowak, G. G. Pooley, K. Pottschmidt, J. S. Sanders, R. R. Ross, and J. Wilms, Mon. Not. R. Astron. Soc. Lett. 424, 217 (2012).

[45] L. Gou, J. E. McClintock, M. J. Reid, J. A. Orosz, J. F. Steiner, R. Narayan, J. Xiang, R. A. Remillard, K. A. Arnaud, and S. W. Davis, Astrophys. J. 742, 85 (2011).

[46] J. E. McClintock, R. Narayan, S. W. Davis, L. Gou, A. Kulkarni, J. A. Orosz, R. F. Penna, R. A. Remillard, and J. F. Steiner, Classical Quant. Grav. 28, 114009 (2011).

[47] C. W. Misner, K. S. Thorne, and J. A. Wheeler, Gravitation (W. H. Freeman and Company, San Francisco, 1973).

[48] S. Privitera, S. R. P. Mohapatra, P. Ajith, K. Cannon, N. Fotopoulos, M. A. Frei, C. Hanna, A. J. Weinstein, and J. T. Whelan, Phys. Rev. D 89, 024003 (2014).

[49] I. W. Harry, A. H. Nitz, D. A. Brown, A. P. Lundgren, E. Ochsner, and D. Keppel, Phys. Rev. D 89, 024010 (2014).

[50] T. A. Apostolatos, Phys. Rev. D 52, 605 (1995).

[51] aligo sensitivity projections for o2, https://dcc.ligo.org/ LIGO-T1600302 (2017), dcc link.

[52] P. Ajith, N. Fotopoulos, S. Privitera, A. Neunzert, N. Mazumder, and A. J. Weinstein, Phys. Rev. D 89, 084041 (2014).

[53] A. Bohé, L. Shao, A. Taracchini et al., Phys. Rev. D 95, 044028 (2017).

[54] A. Buonanno, B. R. Iyer, E. Ochsner, Y. Pan, and B. S. Sathyaprakash, Phys. Rev. D 80, 084043 (2009).

[55] D. A. Brown, I. Harry, A. Lundgren, and A. H. Nitz, Phys. Rev. D 86, 084017 (2012).

[56] I. W. Harry, B. Allen, and B. S. Sathyaprakash, Phys. Rev. D 80, 104014 (2009).

[57] Y. Pan, A. Buonanno, M. Boyle, L. T. Buchman, L. E. Kidder, H. P. Pfeiffer, and M. A. Scheel, Phys. Rev. D 84, 124052 (2011).

[58] S. Marsat, A. Bohé, L. Blanchet, and A. Buonanno, Classical Quant. Grav. 31, 025023 (2014).

[59] M. Hannam, P. Schmidt, A. Bohé, L. Haegel, S. Husa, F. Ohme, G. Pratten, and M. Pürrer, Phys. Rev. Lett. 113, 151101 (2014).

[60] M Pürrer, Classical Quant. Grav. 31, 195010 (2014).

[61] LIGO Scientific Collaboration 2018, LIGO Algorithm Library-LALSuite, Free Software (GPL), https://doi.org/ 10.7935/GT1W-FZ16.

[62] J. D. Hunter, 2007, CSE, 9, 90, https://doi.org/10.1109/ MCSE.2007.55. 